\title{
TOTALLY COMPLEX SUBMANIFOLDS OF THE CAYLEY PROJECTIVE PLANE
}

\author{
by LIU XIMIN
}

(Received 30 July, 1996)

\begin{abstract}
Let $h$ be the second fundamental form of a compact submanifold $M$ of the Cayley projective plane $C a P^{2}$. We determine all compact totally complex submanifolds of complex dimension $n$ in $C a P^{2}$ satisfying $|h|^{2} \leq n$.
\end{abstract}

1. Introduction. Let $M$ be an $n$-dimensional compact Kaehler submanifold of the complex projective space $C P^{m}(1)$. Denote by $h$ the second fundamental form of $M$ and $U M$ the unit tangent bundle over $M$. Ros showed in [5] that if $f(u)=|h(u, u)|^{2}<\frac{1}{4}$ for any $u \in U M$, then $M$ is totally geodesic. Moreover in [6], Ros gave a complete list of compact Kaehler submanifolds of $C P^{m}(1)$ satisfying the condition $\max _{u \in U M} f(u)=\frac{1}{4}$. The same type results for totally complex submanifolds of the quaternion projective space $H P^{m}(1)$ were obtained by Coulton and Gauchman [3]. In [4], Coulton and Glazebrook proved the analogous results in the case of totally complex submanifolds of the Cayley projective place $\mathrm{CaP}$. In the present paper, we proved the following pinching theorem for the square of the norm of the second fundamental form.

TheOREM. Let $M$ be a compact complex submanifold of complex dimension $n$ immersed in Cayley projective plane CaP $P^{2}$. If the square of the norm of the second fundamental form of $M$ satisfies $|h|^{2} \leq n$, then either (i) or (ii) holds.

(i) $|h|^{2}=0, M$ is totally geodesic in $C a P^{2}$, and $M$ is $C P^{1}(1)$ or $C P^{2}(1)$.

(ii) $|h|^{2}=n$ and $M$ is $C P^{1}\left(\frac{1}{2}\right)$.

2. Cayley projective plane. In this section, we review the fundamental results about the Cayley projective plane; for details see [4].

Let us denote by $\mathrm{Ca}$ the set of Cayley numbers, It possesses a multiplicative identity 1 and a positive definite bilinear form $<$, > with norm $\|a\|=<a, a>$ satisfying $\|a b\|=\|a\| \bullet\|b\|$, for $a, b \in C a$. Every element $a \in C a$ can be expressed in the form $a=a_{0} 1+a_{1}$ with $a_{0} \in R$ and $\left\langle a_{1}, 1\right\rangle=0$. The conjugation map $a \rightarrow a^{*}=a_{0} 1-a_{1}$ is an anti-automorphism $(a b)^{*}=b^{*} a^{*}$.

A canonical basis for $C a$ is any basis of the form $\left\{1, e_{0}, e_{1}, \ldots, e_{6}\right\}$ satisfying: (i) $<e_{1}, 1>=0$; (ii) $\left\langle e_{i}, e_{j}\right\rangle=\{0$ for $i \neq j$, and 1 otherwise $\}$; (iii) $e_{i}^{2}=-1 ; \quad e_{i} e_{j}+e_{j} e_{i}=0(i \neq j)$; (iv) $e_{i} e_{i+1}=e_{i+3}$ for $i \in Z_{7}$.

Let $V$ be a vector space of real dimension 16 with automorphism group $\operatorname{Spin}(9)$. The splitting

$$
V=C a \oplus C a
$$

Glasgow Math. J. 40 (1998) 161-166. 
together with the above canonical basis on each summand, endows $V$ with what we refer to as a Cayley structure. We know that the Cayley projective plane $\mathrm{CaP}$ is a 16-dimensional Riemannian symmetric space whose tangent space admits the Cayley structure pointwise. In the following, Let $\left\{I_{0}, \ldots, I_{6}\right\}$ be the Cayley structure on $\mathrm{CaP}^{2}$.

The curvature tensor $\bar{R}$ of $\mathrm{Ca} P^{2}$ is given in [2] as follows

$$
\begin{aligned}
\bar{R}((a, b),(c, d))(e, f)= & \frac{1}{4}\left(\left(<c, e>a-4<a, e>c+(e d) b^{*}-(e b) d^{*}\right.\right. \\
& \left.+(a d-c b) f^{*}\right),(4<d, f>b-4<b, f>d \\
& \left.\left.+a^{*}(c f)-c^{*}(a f)+e^{*}(a d-c b)\right)\right)
\end{aligned}
$$

On $\mathrm{Ca} \oplus \mathrm{Ca}$ we have the positive definite bilinear form $<$, > given by

$$
\langle(a, b),(c, d)\rangle=\langle a, c\rangle+\langle b, d\rangle
$$

3. Totally complex submanifolds. Let $V \subset T_{x} C a P^{2}$ be a real vector subspace, we say that $V$ is a totally complex subspace if there exists an $I$ such that there is a basis with $I=I_{0}$ and (i) $I_{0} \subset V$, and (ii) $I_{k} V$ is perpendicular to $V$ for $1 \leq k \leq 6$. Clearly, if $V$ is a maximal subspace of this kind, then $\operatorname{dim}_{R} V=4$.

Let $M$ be a compact Riemannian manifold isometrically immersed in $\mathrm{CaP}^{2}$ by $j: M \rightarrow C a P^{2}$. Denote by $h$ and $A$ the second fundamental form of $j$ and the Weingarten endomorphism respectively. Then we have

$$
<h(X, Y), N>=\left\langle X, A_{N} Y>\right.
$$

where $X, Y \in T M, N \in T M^{\perp}$. We take $\bar{\nabla}, \nabla$ and $\nabla^{\perp}$ to be respectively the Riemannian connections on $\mathrm{CaP}^{2}, M$ and the normal connection on $M$. The corresponding curvature tensors are denoted by $\bar{R}, R$, and $R^{\perp}$, respectively. The first and second covariant derivatives of $h$ are given by

$$
\begin{aligned}
(\bar{\nabla} h)(X, Y, Z)= & \nabla \frac{1}{Z}\left(h(X, Y)-h\left(\nabla_{Z} X, Y\right)-h(X, \nabla Z Y),\right. \\
\left(\bar{\nabla}^{2} h\right)(X, Y, Z, W)= & \nabla_{W}^{\perp}(\bar{\nabla} h)(X, Y, Z)-(\bar{\nabla} h)(\nabla W X, Y, Z) \\
& -(\bar{\nabla} h)\left(X, \nabla{ }_{W} Y, Z\right)-(\bar{\nabla} h)\left(X, Y, \nabla_{W} Z\right),
\end{aligned}
$$

where $X, Y, Z, W \in T M$. The Codazzi equation takes the following form

$$
(\bar{\nabla} h)\left(X_{r(1)}, X_{r(2)}, X_{r(3)}\right)=(\bar{\nabla} h)\left(X_{1}, X_{2}, X_{3}\right),
$$

where $r \in S_{3}$, the permutation group, and the arguments are in the tangent space of $M$. Recalling that $h$ and $\bar{\nabla} h$ are symmetric, we have the Ricci identity

$$
\begin{aligned}
\left(\bar{\nabla}^{2} h\right)(X, Y, Z, W)-\left(\bar{\nabla}^{2} h\right)(X, Y, W, Z)= & -R^{\perp}(Z, W) h(X, Y) \\
& +h(R(Z, W) X, Y)+h(X, R(Z, W) Y) .
\end{aligned}
$$


We say that $j: M \rightarrow C a P^{2}$ is a totally complex immersion if $W=j_{*}(T M)$ is a totally complex subspace for each point of $M$. Observe that every totally complex submanifold of $\mathrm{CaP} P^{2}$ has a Kaehler structure. We set $I=I_{0}$, and consequently we have

$$
\begin{aligned}
& \text { (a) } \bar{\nabla}_{X} I=0, \\
& \text { (b) } h(I X, Y)=I h(X, Y), \\
& \text { (c) } A_{I N}=I A_{N}=-A_{N} I, \\
& \text { (d) } I R(X, I X) X=R(X, I X) I X
\end{aligned}
$$

where $X, Y \in T_{x} M$ and $N \in T_{x} M^{\perp}$.

Define $f(u)=|h(u, u)|^{2}$, where $u \in U M$, the unit tangent bundle over $M$. Assume $f$ attains its maximum at some vector $v \in U M_{p}$, then by [5] we have

$$
A_{h(v, v)} v=|h(v, v)|^{2} v
$$

Lemma 3.1. Let $M_{n}$ be a compact totally complex submanifold in CaP $P^{2}$. Assume $f$ attains its maximum at $v \in U M_{p}$, then

$$
\left.3|h(v, v)|^{2}\left(1-4|h(v, v)|^{2}+\sum_{i=1}^{6}<h(v, v), I_{i} v>^{2}+4 \mid \bar{\nabla} h\right) v, v, v\right)\left.\right|^{2} \leq 0 .
$$

Proof. Fix $v$ in $U M_{p}$. For any $u \in U M_{p}$, let $r_{u}(t)$ be the geodesic in $M$ satisfying the initial conditions $r_{u}(0)=p, r_{u}^{\prime}(0)=u$. Parallel translating along $r_{u}(t)$ gives rise to a vector field $V_{u}(t)$. Put $f_{u}(t)=f\left(V_{u}(t)\right)$, then

$$
\frac{d^{2}}{d t^{2}} f_{u}(0)=2<\left(\bar{\nabla}^{2} h\right)(u, u, v, v), h(v, v)>+2|(\bar{\nabla} h)(u, v, v)|^{2}
$$

Using (6), (7) and (8), we have

$$
\begin{aligned}
<\left(\bar{\nabla}^{2} h\right)(I v, I v, v, v), h(v, v)>= & <\left(\bar{\nabla}^{2} h\right)(I v, v, I v, v), h(v, v)> \\
= & -<\left(\bar{\nabla}^{2} h\right)(v, v, v, v), h(v, v)>+<R^{\perp}(I v, v) h(I v, v), h(v, v)> \\
& -2<R(I v, v) I v, A_{h(v, v)} v>.
\end{aligned}
$$

From the Ricci equation, (1), (2) and (8), we obtain

$$
\begin{aligned}
<R^{\perp}(I v, v) h(I v, v), h(v, v)> & =<\bar{R}(I v, v) h(I v, v), h(v, v)>+<\left[A_{h(I v, v)}, A_{h(v, v)}\right] I v, v> \\
& =-\frac{1}{2}|h(v, v)|^{2}-\left.2 h(v, v)\right|^{2}+\frac{1}{2} \sum_{i=1}^{6}<h(v, v), I_{i} v>^{2} .
\end{aligned}
$$


Now, by the Gauss equation and using (1), (2) and (8), we have

$$
<R(I v, v) I v, A_{h(v, v)} v>=-|h(v, v)|^{2}+2\left|A_{h(v, v)} v\right|^{2} .
$$

Since $f$ attains its maximum at $v$, we have

$$
\frac{d^{2}}{d t^{2}} f_{v}(0)+\frac{d^{2}}{d t^{2}} f_{I v}(0) \leq 0
$$

Combining (11)-(15) and noticing (9), we get (10).

Lemma 3.2. Let $M$ be a compact totally complex submanifold in $\mathrm{CaP} P^{2}$. Assume fattains its maximum at $v \in U M_{p}$, then for any $u \in U M_{p}$ with $\langle u, v\rangle=\langle u, I v\rangle=0$, we have

$$
|h(v, v)|^{2}\left(1-8|h(u, v)|^{2}\right)-\left|A_{h(v, v)} u\right|^{2}+\sum_{i=1}^{6}<h(v, v), I_{i} u>^{2}+4|(\bar{\nabla} h)(u, v, v)|^{2} \leq 0 .
$$

Proof. Suppose $u \in U M_{p}$ such that $\langle u, v>=<u, I v>=0$. From (7), (8), (11) and the fact that $f$ attains its maximum at $v$, we have

$$
\begin{aligned}
0 \geq \frac{1}{2}\left(\frac{d^{2}}{d t^{2}} f_{u}(0)+\frac{d^{2}}{d t^{2}} f_{l u}(0)\right)= & \left(\bar{\nabla}^{2} h\right)(u, u, v, v), h(v, v)> \\
& +<\left(\bar{\nabla}^{2} h\right)(I u, I u, v, v), h(v, v)>+2|(\bar{\nabla} h)(u, v, v)|^{2} \\
= & <R^{\perp}(I u, u) h(I v, v), h(v, v)>-2<R(I u, u) I v, A_{h(v, v)} v> \\
& +2|(\bar{\nabla} h)(u, v, v)|^{2} .
\end{aligned}
$$

Using the Ricci equation, (1), (2), (8) and (9), we get

$$
<R^{\perp}(I u, u) h(I v, v), h(v, v)>=-\frac{1}{2}|h(v, v)|^{2}-\left|A_{h(v, v)} u\right|^{2}+\sum_{i=1}^{6}<h(v, v), I_{i} u>^{2} .
$$

From the Gauss equation, (1), (2), (8) and (9), we have

$$
-2<R(I u, u) I v, A_{h(v, v)} v>=|h(v, v)|^{2}-4|h(v, v)|^{2}|h(u, v)|^{2} .
$$

From above equations, we get (16).

4. Proof of the Theorem. When $n=1$, it follows easily from $|h| \leq 1$ that $f \leq \frac{1}{4}$, and the conclusion of Theorem is the consequence of Theorem 2.2 in [4]. So we need to consider the case $n>1$. Assume the function $f$ attains its maximum at $v \in U M_{p}$. If $f(v)=0$, then $M$ is totally geodesic. If $f(v) \neq 0$, we want to show that $f(v) \leq \frac{1}{4}$. To do this, let 
$e_{1}, e_{2}=I e_{1}, \ldots, e_{2 n-1}, e_{2 n}=I e_{2 n-1}$ be an orthonormal basis of $T_{p} M$. By the assumption of Theorem, we have

$$
n \geq \sum_{i, j=1}^{2 n}\left|h\left(e_{i}, e_{j}\right)\right|^{2}=4|h(v, v)|^{2}+4 \sum_{i=3}^{2 n}\left|h\left(v, e_{i}\right)\right|^{2}+\sum_{i, j=3}^{2 n}\left|h\left(e_{i}, e_{j}\right)\right|^{2} .
$$

From (9), we know that $A_{h(v, v)} v \backslash \backslash v$ and $A_{h(v, v)} I v \backslash \backslash I v$. Thus, for $i \geq 3$, we have

$$
<A_{h\left(e_{1}, e_{1}\right)} e_{i}, e_{1}>=<A_{h\left(e_{1}, e_{2}\right)} e_{2}, e_{i}>=0,
$$

and so when $i \geq 3$,

$$
\begin{aligned}
& \sum_{j=3}^{2 n}\left|h\left(e_{i}, e_{j}\right)\right|^{2} \geq \sum_{j=3}^{2 n}\left(<h\left(e_{i}, e_{j}\right), \frac{h(v, v)}{|h(v, v)|}>^{2}+<h\left(e_{i}, e_{j}\right), \frac{I h(v, v)}{|\operatorname{Ih}(v, v)|}>^{2}\right) \\
& =\frac{2}{|h(v, v)|^{2}} \sum_{j=3}^{2 n}<h\left(e_{i}, e_{j}\right), h(v, v)>^{2}=\frac{2}{|h(v, v)|^{2}} \sum_{j=3}^{2 n}<A_{h(v, v)} e_{i}, e_{j}>^{2} \\
& =\frac{2}{|h(v, v)|^{2}} \sum_{j=3}^{2 n}<A_{h(v, v)} e_{i}, e_{j}>^{2}=\frac{2}{|h(v, v)|^{2}} \sum_{j=3}^{2 n}\left|A_{h(v, v)} e_{i}\right|^{2} .
\end{aligned}
$$

Also, when $i \geq 3$, we have by Lemma 3.2

$$
1-8\left|h\left(v, e_{i}\right)\right|^{2}-\frac{4}{|h(v, v)|^{2}}\left|A_{h(v, v)} e_{i}\right|^{2} \leq 0 .
$$

From the above equations, we obtain

$$
\begin{aligned}
n & \geq 4|h(v, v)|^{2}+\sum_{j=3}^{2 n}\left(4\left|h\left(v, e_{i}\right)\right|^{2}+\frac{2}{|h(v, v)|^{2}}\left|A_{h(v, v)} e_{i}\right|^{2}\right) \\
& \geq 4|h(v, v)|^{2}+\frac{2 n-2}{2} .
\end{aligned}
$$

Thus $f(u) \leq \frac{1}{4}$ for any $u \in U M$. The theorem follows from Theorem 2.2 in [4]. This completes the proof of the theorem.

ACKNowledgement. The author would like to thank the referee for his helpful suggestions.

\section{REFERENCES}

1. A. L. Besse, Manifolds all of whose geodesics are closed (Springer-Verlag, Berlin, 1978).

2. R. Brown and A. Gray, Riemannian manifolds with holonomy group Spin (9), in Diff. Geom. in honor of K. Yano, 42-59 (Tokyo, 1972). 
3. P. Coulton and H. Gauchman, Submanifolds of quaternion projective space with bounded second fundamental form, Kodai Math. J. 12 (1989), 296-307.

4. P. Coulton and J. Glazebrook, Submanifolds of Cayley projective plane with bounded second fundamental form, Geom. Dedi. 33 (1990), 265-272.

5. A. Ros, Positively curved Kaehler submanifolds, Proc. Amer. Math. Soc. 93 (1985), 329-331.

6. A. Ros, A characterization of seven compact Kaehler submanifolds by holomorphic pinching, Ann. of Math. 121 (1985), 377-382.

7. K. Tsukada, Parallel submanifolds in a quaternion projective space, Osaka J. Math. 22 (1985), $187-241$.

Department of Mathematics

NANKaI UNIVERSITY

TIANJIN 300071

P. R. China
Present address:

Department of Applied Mathematics

Dalian University of TeChNOLOGy

DALIAN 116024

P. R. China 